\title{
Medical Education on Alcohol Dependence: The Relative Impact of Cognitive, Attitudinal and Clinical Approaches*
}

\author{
Marcus Grant, Director, Alcohol Education Centre, Maudsley Hospital, London
}

\begin{abstract}
Increasing attention continues to be paid to the discrepancy between the current performance of medical practitioners in dealing with alcohol dependence and the enormous potential which exists for early diagnosis and cost-effective treatment. This discrepancy has been noted in various government reports, in articles in the medical press and in the seminal report of the Royal College of Psychiatrists, Alcohol and Alcoholism. One way of narrowing the gap between performance and potential would be through improvements in medical education. The purpose of this short paper is to review existing evaluated studies of medical education on alcohol dependence in order to discover whether any specific lessons emerge which might be useful in planning curriculum changes either at undergraduate or postgraduate level.
\end{abstract}

There is strong evidence to support the view that many medical schools devote very much less time and attention to this topic than its real clinical importance would require. Alcoholics are not, of course, popular with either physicians or psychiatrists, but it is difficult to determine whether their lack of popularity is the result of negative clinical experience or of inadequate and ill-informed teaching.

Within the period 1960-1980 only ten studies were identified which reported upon specific alcohol education programmes within the medical curriculum. One further study carried out by the American Career Teacher Scheme will be discussed separately. (These 11 studies are listed as the references to this paper.) It may well be that much medical education on this topic is not evaluated or, having been evaluated, remains unpublished. Within this review, a programme is described as evaluated when some attempt has been made to measure its impact upon knowledge, attitudes or clinical skills. Whilst subjective ratings of course content do not constitute evaluation for these purposes, it should be recognized that none of the studies in this review adequately matched the requirements of rigorous experimental methodology. The best that can be said is that most of them tried to use some kind of comparison group and to obtain pre-course and post-course measures for the variable(s) in which they were interested.

\section{Educating against pessimism}

Of the ten studies in this review, nine were undertaken in the United States and one in England. The nine US studies were all concerned with alcohol education in the undergraduate curriculum (during the first three years of medical - This article is based on a paper presented at the Winter Quarterly Meeting of the Colloge
in November 1981 . school) or during the period of psychiatric residency. The one English study considered the response of general practitioners to a multidisciplinary training course.

The information provided in these studies about the nature and content of the alcohol education is not sufficiently detailed to enable any useful analysis to be made of what exactly was being taught. It is, however, possible to say something about the teaching approach, particularly in terms of the relative emphasis placed upon cognitive, attitudinal and clinical aspects of the educational experience.

Virtually every paper reviewed began by noting how negative the attitudes of medical practitioners are towards alcohol dependent patients. There appeared to be some evidence (Fisher et al, 1975) that medical students became more negative towards such patients as their training progressed and that they certainly emerged from medical school with lower expectations of them than they had when they first entered. These negative attitudes are likely to undermine doctors' motivation to treat alcohol dependent patients. Equally, their sense of therapeutic pessimism will generalize so as to limit even further the possibilities of successful treatment outcomes. Unless confronted directly, therefore, this pessimism is likely to take on the characteristics of a selffulfilling prophesy in which each relapse confirms how right the doctor was never to hope for a better prognosis.

Pessimism also has its impact upon the training process itself. Nocks (1980) found that: 'Negative attitudes in students seem to impair the acquisition of knowledge, and, as a corollary, factual material which is learned often does not improve the physician's performance with alcoholic patients, since these negative attitudes typically persist.' The implication that a wholly or substantially cognitive approach to alcohol education in the medical curriculum is unlikely to achieve optimum impact is supported by the authors of other studies. Indeed, one study recommends a bespoke approach to this educational problem rather than the current off-thepeg style of curriculum development. 'Concentrating on imparting information about alcoholism,' suggested Fisher et al (1976), 'with the implicit assumption that greater knowledge will lead to better attitudes, may not be sufficient to alter attitudes favourably. Instead, the probability of attitudinal change might be enhanced by surveying attitudes prior to training and then designing the curriculum around the focal issues that require modification.'

That factual information is currently being communicated or, at least, that doctors believe themselves to have sufficient knowledge to be able to undertake treatment, is supported by the UK study (Cartwright, 1980) which 
found that doctors were less anxious than social workers and probation officers about the state of their clinical knowledge. What remains, therefore, is the problem of how to structure course work so as to encourage a more optimistic prognosis for alcohol dependence which is nevertheless not at odds with the reality of clinical experience, which is likely to bring with it its fair share of relapses. It may, therefore, be important to take into account both the extent to which alcohol dependence can legitimately be perceived as a selfinflicted condition and also the role of the doctor in contributing to a process of apparent spontaneous remission which may be more frequent than tends to be acknowledged.

At its most basic, what is required is some kind of demonstration that alcohol dependence is treatable. Such a demonstration, of little value in itself, may clear the way for substantial clinical training and ensure that alcohol dependence is seen as worthwhile from a clinical standpoint. There are implications here for course design. Chodorkoff (1967) notes that: 'A program designed to give clinical experience must teach an approach to the patient in addition to providing specific information or knowledge about clinical entities and theoretical issues.' Like many other members of the general population, medical students tend to see alcoholism* as a product of weak will and foolishness (Fisher et al, 1976). This view appears to be particularly strong amongst the more authoritarian students (Chodorkoff, 1967). Nevertheless, educational approaches have the capacity (Fisher $e t$ al, 1976) to enlarge the concept of legitimate sickness.

What needs to be recognized are the inappropriate assumptions which the sick role sometimes confers upon people, such as lack of responsibility for participating actively in the recovery process, and the increased potential which properly trained doctors would have for optimizing their unparalleled opportunities for therapeutic intervention. The studies in this review have tended to look at alcohol dependence from an individual patient perspective but they also have implications for a wider public health approach, for which something more than sharpened clinical skills are going to be needed. It is not enough, therefore, just 'to make doctors better at treating alcoholics'. Their positive attitudes must also embrace a substantially increased preventive effort which can find a focus in general hospitals and in general practitioners' surgeries as well as in psychiatric clinics.

\section{Impact upon knowledge, attitudes or clinical skills}

The most encouraging aspect of this review is the extent to which medical education on alcohol dependence emerges as having the potential to produce positive impact upon its The US studies all tend to presume the validity of 'alcoholism' as a discrete illness. This tendency is reflected in extracts from these studies and in comments upon them. The term 'alcohol dependence' as used in the body of the present paper is intended to refer to a wider concept. No attempt has been made here to distinguish the many different ways in which excessive drinking may impinge upon clinical practice. recipients. The author of this paper is currently reviewing all evaluated alcohol education programmes (including school education, mass media education and professional training) published in North America and Western Europe between 1960 and 1980. The ten medical education studies reported upon here comprise only about 5 per cent of the total number of evaluated alcohol education programmes of all categories, yet they represent what may well prove, upon further analysis, to be the highest proportion of positive results, particularly in the areas of attitudes and behaviour (in this case, usually clinical skills).

No alcohol education programme in this or in any other category has actually been shown to diminish or to fail to increase knowledge, although not every evaluation tested for this variable. It does seem, however, that those who sought to increase knowledge about alcohol and alcohol dependence have found this a relatively simple goal to achieve. Difficulties more frequently occur when attempts are made to change attitudes or behaviour. In fact, the ten studies in the present review display heartening results in both those areas. The percentage of positive results is far higher than has been achieved either by mass media programmes directed towards the general population or by alcohol education programmes directed towards young people.

The reasons for the relative success of professional education in this area are not difficult to determine. People who have chosen to enter a profession which includes responding to alcohol problems are more likely to have a high motivation towards alcohol education than people who do not perceive alcohol problems as being relevant to their own lives. Further, people who perceive their professional responsibilities in terms of helping others are likely to be influenced positively by education and training designed to improve the nature of the help which they are able to offer. Whilst the odds may be stacked against success with respect to mass media approaches to alcohol education, they are stacked in favour of success with respect to professional training.

Analysis of the studies in this review also suggests that programmes which adopt a strong attitudinal and clinical approach stand a very good chance of making positive impact in those very areas. Since the potentially damaging effects of negative attitudes amongst doctors is so considerable and is likely to influence both susceptibility to further training and likelihood of future clinical involvement with alcohol dependent patients, the importance of this finding is very considerable. It suggests that medical education has the capacity to confront therapeutic pessimism head-on and to make substantial impact upon pervading negative attitudes, thus opening the door to clinical training and subsequent clinical involvement.

Fisher et als (1975) examination of the impact of the normal undergraduate medical curriculum supports such a view. They conclude that: 'The results of the present study suggest that attitudinal education would have optimal 
chance of success if it involved clinical training and taught proper attitudes in the same manner as informational matters are currently taught.' Whilst the actual wording of that conclusion does seem to beg a number of questions, particularly in relation to the methods of communication selected for different educational objectives, it does serve to underline the importance of keeping in mind an integrated model of the medical curriculum in which the threads of cognitive and clinical approaches are woven together in a single experience.

Similarly, even a study (Chodorkoff, 1967) reporting no significant attitude change, felt able to assert: 'We could predict from the individual changes that occurred that the more change we produced (in attitudes) the more we could expect the student to learn from the clinical and teaching experiences.' In general, therefore, these examples support the view that it is possible to design components of the undergraduate (and possibly of the postgraduate) medical curriculum which will ensure that doctors know more about alcohol problems, feel better about dealing with alcohol dependent patients and have a more optimistic expectation of the outcome of their intervention.

It is, inevitably, much more difficult to make unequivocal statements about the impact of education upon behaviour (clinical skills). Those studies reporting positive behavioural impact used diagnosis rates as their criterion for success. This has the advantage of being something which is measurable and which is relatively objective. It says nothing, however, about what happens to the patients once they have been diagnosed and whether the specially trained doctors were better able to help them with their alcohol problems. Methodologically, there are huge difficulties in taking the evaluations beyond diagnosis into treatment. It may, however, be possible to go some way along that road if, instead of looking beyond the rainbow for treatment outcome rates, attempts are made to look at the treatment process and the extent to which the reduction of therapeutic pessimism may have helped to let fresh breezes blow through the windows of a few alcoholism clinics.

There is a further variable which only one study (Hart, 1979) had the courage to measure. That study found, as the result of the alcohol education offered to first year medical students, no reported change in personal drinking behaviour.

\section{Conctusions}

The same study (Hart, 1979) which looked at personal drinking behaviour also recognized where the responsibility lies in stimulating the necessary changes in the curriculum. It states: 'First year medical students clearly want training in alcoholism. While they may not initiate the training, if it is offered they avail themselves of the opportunity to partake of it. Thus the burden must fall upon the responsible faculty to put forth the efforts necessary to create, fund and carry out student training in the area of alcoholism.'

The pioneering Career Teachers Scheme in the US repre- sents an important demonstration of what can indeed be achieved by faculty members provided they have the commitment and the opportunity to influence the curriculum. Commitment is present in many places where opportunity is sadly limited. It needs to be recognized that providing the opportunity to develop alcohol education within medical training means a great deal more than asking an already overburdened consultant psychiatrist to take on yet another responsibility. It involves making this task an explicit part of the continuing teaching responsibility of tenured faculty members. It involves relieving them of other duties in order to permit them to do the task justice. It involves, in other words, putting our money where our mouth is.

The evaluation of the Career Teachers Scheme (CONSAD, 1977) indicates how significant the impact of such an exercise may be. It is difficult, as noted above, to be sure of long-term changes in clinical practice. To use a very simple and immediate measure, however, the report notes that: 'There has been an average increase in curriculum hours (devoted to alcohol education in medical schools operating the Career Teachers Scheme) from 18.5 to 123.6 hours, of which almost all is attributable to Career Teachers.' Equally important in the long term is the effect of such initiatives on the medical schools themselves. Given the pervasiveness of the therapeutic pessimism which has already been noted, it would be reasonable to assume that the soil of most medical schools appeared relatively barren for the sowing of the first Career Teachers' educational seeds. It seems, however, that 25 medical schools in the US are now reporting that there is an increase in the number of faculties voluntarily involving themselves in the teaching of substance abuse at different points in the curriculum.

It is unrealistic to suggest that the Career Teachers Scheme can simply be replicated in other countries. Quite apart from its prohibitively high costs (over $\$ 5$ million to date), it presumes particular structures of influence both within medical schools and between medical schools, universities and central government which are far from identical in different parts of the world. What the scheme demonstrates, however, is that it is possible to make an impact on the curriculum, however inflexible and constraining it may seem at first sight. Just as unreasonable pessimism clouds the vision of the emerging medical student with regard to alcohol dependence, so that same unreasonable pessimism sometimes prevents those interested in stimulating alcohol education from taking a few bold steps along a path which clearly leads in the right direction. There is little point in pleading lack of funds, lack of motivation and lack of sympathetic response from colleagues. Such impediments are the burden that innovators always have to bear and are, indeed, a way of testing the centrality, the rightness, the precision of the suggested changes. They are challenges to be welcomed rather than barriers which have to be scaled with weary reluctance.

Part of the frustration in dealing with alcohol dependent 
patients may well relate to the perfectly understandable desire of clinicians to be able to be of more help. Coming to terms with a set of realistic clinical expectations is probably the best cure for therapeutic pessimism. That same lesson may apply to attempts to change the medical curriculum. A few modest improvements may not seem to produce the radical revision that the inertia of existing practice cries out for; but these same modest improvements may have an influence that outstrips their immediate impact. The trick is to be sure that you are moving in the right direction. Then even the smallest step is an important advance.

\section{ACKNOWLEDGEMENTS}

Special thanks are due to the Alcohol Research Group, School of Public Health, University of California, Berkeley, and to the Social Aspects of Alcohol Committee of the Wine and Spirit Association of Great Britain.

\section{BIBLIOGRAPHY}

Cartwright, A. K. J. (1980) The attitudes of helping agents towards the alcoholic client: The influence of experience, support, training and self-esteem. British Journal of Addiction, 75, 413-31.

Chappel, J. N., Jodan, R. D., Treadway, B. J. \& Miller, P. R. (1977) Substance abuse attitude change in medical students. American Journal of Psychiatry, 134, 379-84.

Снodorkoff, B. (1967) Alcoholism education in a psychiatric institute: I. Medical students: relationship of personal characteristics, attitudes towards alcoholism and achievement. Quarterly Journal of Studies on Alcohol, 28, (4), 72330.

CONSAD Research Corporation (1977) Career Teacher Program Evaluation Vols. I, II, III, Consad Research Corporation, Pittsburgh, Pennsylvania.

Fisher, J. C., MAson, R. L., Keeley, K. A. \& Fisher, J. V. (1975) Physicians and alcoholics: The effect of medical training on attitudes toward alcoholics. Journal of Studies on Alcohol, 36, (7), 949-55.

Fisher, J. V, Fisher, J. C. \& MAson, R. L. (1976) Physicians and alcoholics: Modifying behaviour and attitudes of family practice residents. Journal of Studies on Alcohol, 37, (11), 1686-93.

Gross, G. A. (1976) Training the medical student in alcoholism therapy. Annals of the New York Academy of Science, 273, 433-35.

HART, J. L. (1979) Alcoholism Training Impact on First Year Medical Students. Ph.D. Thesis, Ohio State University.

Nocks, J. J. (1980) Instructing medical students on alcoholism: What to teach with limited time. Journal of Medical Educa tion, 55, (10), 858-64.

PACE, N. A. (1977) The Cornall medical students' field trip through the world of alcoholism. In Currents in Alcoholism: Psychiatric, Psychological, Social and Epidemiological Studies, ed. F. A. Seixas. New York: Grune and Stratton.

WeInBerG, J. R. \& Morse, R. M. (1976) Understanding alcoholism: a test for use in medical education. Alcohol Health and Research World, 1, 16-17.

\section{News Items}

\section{The Burden Research Gold Medal and Prize}

The Burden Research Gold Medal and Prize for outstanding published research work in the field of mental handicap during the past three years was presented on 7 June 1982 at Stoke Park Hospital, Bristol, to Dr J. A. Corbett, Consultant Psychiatrist, Bethlem Royal and Maudsley Hospitals, London.

\section{The Psychotherapy Section of the Scottish Division}

The Psychotherapy Section of the College's Scottish Division has successfully held residential conferences twice a year for a decade or so. The beauty of Pitlochry, the comfort of Scotland's Hotel and a relatively constant membership provide the therapeutic setting. The variety of people, places, skills and styles represented, and the intensive formal and informal presentations and discussions provide the material and work of the conference.
The last conference (29 April to 1 May 1982) was entitled 'On the Borderline'-a concern for the theorist and the practitioner, for the expert psychotherapist and the novice psychiatrist. Some forty-five people attended, and it is hoped most of them will return to the next conference on 'Group Psychotherapy' to be held at the end of October 1982.

\section{Psychopharmacology for the Trainee Psychiatrist}

The Upjohn Company have produced a book called Introduction to Psychopharmacology written by Professor Malcolm Lader. The book aims to present succinctly the pharmacological basis for the clinical use of psychotropic drugs, and is principally designed for the psychiatrist in training, but general practitioners, psychologists and pharmacologists should find it useful.

Copies are available, free of charge, on request to: Introduction to Psychopharmacology, Upjohn Ltd., Fleming Way, Crawley, West Sussex RH10 2NJ. 